\title{
O retorno à casa: uma revisão sistemática sobre Homeschooling e Autismo
}

\author{
Telma Maria Duarte Rodrigues* \\ Miria Benincasa** \\ Hilda Rosa Capelão Avoglia***
}

\section{Resumo}

A educação doméstica - homeschooling - vem se constituindo em uma preocupação no âmbito da legislação dos direitos assim como para os processos psicoeducativos e a sociedade em geral. A questão se torna ainda mais complexa no contexto específico de pessoas com Transtorno do Espectro Autista (TEA). Assim, o objetivo desse estudo foi discutir a efetividade do bomeschooling e, em especial, no caso de pessoas com TEA. Trata-se de uma pesquisa do tipo bibliográfica sistemática, utilizando desenho metodológico PRISMA. Desse modo, foram usados como descritores: homeschooling, família e autismo, a partir das bases de dados: Bibliotecs Virtual de Saúde (BVS-PSI), SCIELO, Periódicos CAPES e Education Resources Information Center - ERIC, considerando o período de janeiro de 2009 a dezembro de 2019. Os resultados, após o processo de sistematização com uso dos referidos descritores, somaram 6 artigos que apontaram os resultados da pesquisa. Foi possível identificar que, segundo familiares, a escolha dessa estratégia educativa ocorre a partir do fracasso escolar e das dificuldades de inclusão social, sendo mais acentuados no caso de familiares de crianças com TEA. Os resultados apontam tratar-se de uma escolha

* Mestranda da Pós-Graduação em Psicologia da Saúde da Universidade Metodista de São Paulo. Bolsista CAPES.

** Professora Doutora do Pós-Graduação em Psicologia da Saúde da Universidade Metodista de São Paulo e do Programa de Pós-Graduação em Psicossomática da Universidade Ibirapuera.

*** Professora Doutora do Pós-Graduação em Psicologia da Saúde da Universidade Metodista de São Paulo e do Programa de Pós-Graduação em Psicologia, Desenvolvimento e Políticas Públicas de Universidade Católica de Santos. 
de vida que envolve a todos, a falta de conteúdos e ações escolares e o prejuízo nas relações familiares. A análise indicou ainda a necessidade da proposição de políticas públicas subsidiadas cientificamente e pertinentes a realidade da complexidade dessa demanda.

Palavras-chave: Homeschooling; Familiares; Autismo; Transtorno do Espectro Autista (TEA).

\section{Returning Home: A Systematic Review of Ho- meschooling and Autism}

\section{Abstract}

Domestic education - homeschooling - is becoming a concern within the scope of rights legislation as well as for psychoeducational processes and society in general. The issue becomes even more complex in the specific context of people with Autistic Spectrum Disorder (ASD). Thus, the aim of this study was to discuss the effectiveness of homeschooling and, in particular, in the case of people with ASD. This is a systematic bibliographic research, using a PRISMA methodological design. Thus, the following descriptors were used: homeschooling, family and autism, from the databases: Virtual Health Libraries (VHL-PSI), SCIELO, CAPES Periodicals and Education Resources Information Center - ERIC, considering the period of January 2009 to December 2019. The results, after the systematization process using these descriptors, added up to 6 articles that pointed out the results of the research. It was possible to identify that, according to family members, the choice of this educational strategy occurs from school failure and difficulties of social inclusion, being more accentuated in the case of family members of children with ASD. The results indicate that it is a life choice that involves everyone, the lack of school content and actions and the damage to family relationships. The analysis also indicated the need to propose scientifically subsidized public policies that are relevant to the reality of the complexity of this demand.

Key- Words: Homeschooling; Parentes; Autismo.

\section{Regresar a casa: una revisión sistemática de Homeschooling y el autismo}

\section{Resumen}

La educación en el hogar - Homeschooling - se ha convertido en una preocupación dentro del ámbito de la legislación de derechos, así como para los 
procesos psicoeducativos y la sociedad en general. El problema se vuelve aún más complejo en el contexto específico de las personas con trastorno del espectro autista (TEA). Así, el objetivo de este estudio fue discutir la efectividad de la educación en el hogar y, en particular, en el caso de personas con TEA. Se trata de una investigación bibliográfica sistemática, utilizando el diseño metodológico PRISMA. Así, se utilizaron los siguientes descriptores: educación en el hogar, familia y autismo, con base en las siguientes bases de datos: Bibliotecas Virtuales de Salud (BVS-PSI), SCIELO, Centro de Información de Revistas y Recursos Educativos CAPES - ERIC, considerando el período de enero de 2009 a diciembre de 2019. Los resultados, luego del proceso de sistematización utilizando estos descriptores, totalizaron 6 artículos que señalaron los resultados de la investigación. Se pudo identificar que, según familiares, la elección de esta estrategia educativa se basa en el fracaso escolar y las dificultades de inclusión social, siendo más acentuada en el caso de familiares de niños con TEA. Los resultados muestran que esta es una elección de vida que involucra a todos, la falta de contenido y acciones escolares y el daño a las relaciones familiares. El análisis también indicó la necesidad de proponer políticas públicas científicamente subsidiadas relevantes a la realidad de la complejidad de esta demanda.

Keywords: Homeschooling; Parientes; Autismo; Trastorno del Espectro Autista (TEA).

\section{Introdução}

Nos últimos tempo, em relação a educação, no Brasil e no mundo verifica-se uma tendência ao ensino domiciliar, possivelmente devido a uma insatisfação com o sistema de ensino e com a realidade da inclusão social. Esse programa - ensino domiciliar - é conhecido nos Estados Unidos como Homeschooling, sendo esse seu país de origem.

Os dados estáticos nos E.U.A apontam que, desde 1999, há um crescimento desse programa, que inicialmente contava com a participação de 850.000 famílias e atualmente são 4,6 milhões de famílias que aderiam ao Programa National Center for Education Statitics (NCES, 2019). No Brasil, estima-se que existam 7 mil crianças pelo país adotantes desse programa, conforme dados da Associação Nacional de Educação Domiciliar (ENAD, 2019). 
Homeschooling é uma denominação de educação que ocorre no ambiente privado, podendo ocorrer em casa, com a presença de professores contratados ou do próprio familiar que estabelece uma relação de um único professor para o filho. No que se refere a questão das ações pedagógicas, dos conteúdos programáticos e das estratégias para o ensino, estes ficam sob a responsabilidade dos familiares e sem a interferência do Estado (Vasconcelos \& Morgado , 2014). Para Ribeiro (2011), este programa é a educação que vem de casa, a qual as crianças e jovens também passariam menos tempo em casa do que os alunos regulares permanecem na escola; pois a educação na casa engloba inúmeras atividades fora do ambiente doméstico, tais como: idas aos museus, passeios em parques e atividades chamadas extracurriculares.

Em 2019, no Brasil, a educação domiciliar teve prioridade no âmbito do governo federal, uma vez que o Ministério da Mulher, Família e Educação, por meio de seu representante maior, a ministra Damares Alves, falou ${ }^{1}$ que muitos pais entendem que seus filhos não estão se adaptando às escolas e apontam como a melhor solução "ficar em casa". No entanto, em nosso país, esse programa ainda não foi regulamentado, além do que não se encontram possibilidades no texto da legislação devido as omissões, lacunas ou interpretações diversas, que são mais afeitas à experiências educacionais permitidas ou à possibilidade de certificação de estudos supletivos presentes nas leis educacionais. (Vasconcelos \& Morgado, 2014). Desta forma, questiona-se a eficácia desse retorno ao ensino domiciliar para as crianças, mas em especial para as crianças autistas.

Atualmente, o Transtorno do Espectro Autista (TEA) assume um lugar relevante nas clínicas, escolas e na sociedade de modo

Na Revista Gaúcha Atualidade (Blog da cidadania , 2019), em entrevista na data de 12/abril/2019, a ministra Damares Alves: "Os pais das crianças com deficiência, pais de crianças com autismo, esse é um grupo muito grande que tem conversado com esse Ministério, eles gostariam de educar os filhos em casa. Muitos deles entendem que os filhos não estão se adaptando na escola, tem criança com autismo que sofre mais indo para escola do que ficando em casa, então (a proposta) também vem para atender essa parcela significativa da população — disse a ministra". 
geral, devido a um crescente número de diagnósticos proporcionado pela necessidade prevenção e intervenção precoce aos indicadores de risco de TEA em crianças até os 18 meses de idade. Associa-se a isso o número crescente de estudo sobre sua etiologia, história e tratamento, aderindo a estudos multidisciplinar como áreas da psicologia, psicanálise, medicina, fonoaudiologia, educação, entre outros (Portolese, Bordini, Lowenthal, Zachi, \& Silvestre de Paula , 2017).

O termo "autismo", apareceu pela primeira vez pelo psiquiatra Eugen Bleuer como um dos quatros critérios para realizar o diagnóstico da esquizofrenia. Os quatro critérios ficaram conhecidos como "os quatro 'A's de Bleuler: alucinações, afeto desorganizado, incongruência e autismo. Mas, apenas em 1943, com o psiquiatra Leo Kanner, que o autismo foi observado nas crianças, com as seguintes características: "(a) inabilidade em desenvolver relacionamentos com pessoas; (b) atraso na aquisição da linguagem; (c) uso não comunicativo da linguagem após o seu desenvolvimento; (d) tendência à repetição da fala do outro (ecolalia); (e) uso reverso de pronomes; (f) brincadeiras repetitivas e estereotipadas; (g) insistência obsessiva na manutenção da "mesmice" (rotinas rígidas e um padrão restrito de interesses peculiares); (b) falta de imaginação; (i) boa memória mecânica; e (j) aparência física normal' (RUTTER, 1978 apud Saúde, 2019).

A descrição e o conceito de autismo infantil, sofreu diversas modificações desde seu aparecimento, tanto que foi agrupado a um conjunto de descrições que se denominou Transtorno Global (ou Invasivo) do Desenvolvimento (TGD), e recentemente denomina-se Transtorno do Espectro Autista (TEA) incluindo: o Autismo, a Síndrome de Asperger e o Transtorno Global do Desenvolvimento sem outra especificação (não incluí a Síndrome de Rett e o Transtorno Desintegrativo da Infância) (DSM-5, 2013).

O TEA é identificado na infância, principalmente antes dos 5 anos de idade e pode permanecer na adolescência e vida adulta. E os sintomas se apresentam por graus: leve, moderado e grave. Além disso, há uma questão de como na clínica localizamos a singularida- 
de desses sujeitos pela sua história, forma de estar no mundo, e/ou se deixa ou não enlaçar pelo outro (Merlleti, 2018).

Em abril de 2018, o Controle de Doenças e Prevenção de Saúde (CDC, 2019), nos Estados Unidos, lançou um estudo com um aumento de prevalência de 1 para 59 crianças com o diagnóstico de Transtorno do Espectro Autista. No mundo, atualmente, temos a prevalência de 1 para 160 crianças. $O$ aumento sugestivo nos Estados Unidos da América, ocorre pela melhora nas bases de estudos epidemiológico com critérios de diagnóstico e informações obtidas pelo Manual de estática e diagnostico (DSM-5, 2013), dados pelo CDC.

No Brasil, ainda não é possível contar com esses dados em nossas bases de estatísticas na vigilância da saúde. Entretanto, em 2015, ocorreu o decreto de uma lei nacional (lei 13.146), conhecida como Lei Brasileira de Inclusão, que autoriza as pessoas com Transtornos do Espectro Autista (TEA), a ser incluído na comunidade, com todos os direitos igualitários aos deficientes como: vida digna, integridade física e moral, livre desenvolvimento da personalidade, segurança e lazer. Proteção ao abuso e exploração. Na saúde, há acessos a serviços de saúde e equipe multidisciplinar, o diagnóstico é precoce, possuem medicação e nutrição. Direito a educação, cursos profissionalizantes e assistência social. (Brasil, 2019).

Até o século XIX, no Brasil não havia nenhuma estrutura ou sistematização em relação a saúde mental. A mudança aconteceu, após a construção do primeiro hospital psiquiátrico, que levou o nome de Pedro II, em 1852, em comemoração da maioridade do Imperador Dom Pedro II (Ribeiro, 2006). Esse hospital era de modelo francês, tendo ótimos recursos para a época. O Brasil, estava saindo da marca do período colonial, o qual os pacientes que possuíam transtornos mentais eram acolhidos nas Santas Casas, por sacerdotes, curandeiros e não médicos, sendo que as condições sanitárias eram precárias.

A partir dessa construção, cria-se também manicômios e a assistência aos doentes mentais, que passaram a ter regulamentação própria e lugar especifico para tratamento e internação, resultando 
em diversos estudos sobre a doença mental no Brasil. Mas só em 1881, que a psiquiatria se torna uma cadeira na faculdade de medicina da Bahia e Rio de Janeiro, independente da medicina legal, momento que começa o seu nascimento no Brasil. Porém os estudos sobre a psiquiatria infantil e psicologia são mais recentes, tendo uma vertente de equipe multidisciplinar (Ribeiro, 2006).

Entretanto, a maioria das vertentes que se preocupava com as crianças e os cuidados assistenciais eram as iniciativas particulares com características filantrópicas e assistencialistas. As transformações no campo da saúde mental sobre pediatria, higiene e o direito vem mais tarde de modelos dos Estados Unidos da América e Europa.

Diante desses modelos, em 27 de dezembro de 2012, na lei 12.764 (Saúde, 2015) como já dito acima as pessoas com TEA tem direitos e proteção na sociedade e na escola, como: existe um asseguramento que as escolas não podem deixar de acolher essas crianças. Porém, e apesar do crescente número de crianças com TEA no mundo, ainda temos dificuldade de interações e preparo dos profissionais para lidar com essas crianças e elas estarem na sociedade. E é importante lembrar que, ao passar dos anos, os sintomas podem se tornar mais cronificados, as relações se tornam mais empobrecidas e a dificuldade de inclusão na sociedade se torna menos eficaz. Por isso que nos EUA está havendo um aumento de familiares que optam pelo retorno e segurança de seus lares, procurando o programa Homeschooling.

Foi verificado em uma pesquisa qualitativa que a escolha pelo Programa de Homeschool pelos pais que tinham filhos com TEA, era devido a diversas questões como: a compreensão sobre o diagnóstico e tratamento para seus filhos com TEA, já tinham tentando diversos métodos psicológicos a dietas, porém reconheciam que seus filhos acabavam por apresentar um atraso a mais no desenvolvimento e suas terapias fracassavam, como também obtinham uma insatisfação pela educação, pois o ambiente na escola ocasionava uma exclusão aos seus filhos, pois esses eram colocados em grupos com crianças de outros transtornos, ou em salas isola- 
dos. A aprendizagem não obtinha um cunho de interesse, e os pais eram chamados muitas vezes na escola necessitando estar defendo os direitos de seus filhos (Hurlbutt, 2011).

No referido artigo , aparecem diversos pontos negativos e fracasso sobre o estar escola regular, como apresenta Hurlbutt (2011): a) não colocar a criança em sala de aula com os demais, e a cada ano era mais frequente o bullying, além da presença dos filhos estar com crianças mais severas; b) programa educacional fracassado; c) sem apoio em sala de aula; d) falta de implementação de recursos e programas; e o e) stress que era causado na família pois quase todas as semanas eram chamados na escola, prejudicando seus trabalhos.

Para os pais, que se reuniam e estabeleceram metas a serem cumpridas para que fosse mais bem exercido esse programa Homeschool, estipulou-se a necessidade de incluir instrução sobre leitura, artes linguísticas, matemática, estudos sociais e ciências (Simmons, \& Campbell, 2018).

Por isso, para essa pesquisa se questionou o porquê alguns pais retornam as suas casas, deixando a socialização que se encontra na escola e no social. Desta forma, o objetivo desse trabalho foi compreender se o homeschooling é eficaz para pessoas com transtornos do espectro autista. E se esse método pode ser utilizado para todos. O método da pesquisa é quantitativa e sistemática, utilizando a estratégia da metodologia PRISMA (Galvão, Pansani, \& Harrad, 2015).

\section{Método}

O presente estudo foi estruturado conforme a estratégia metodológica Prefered Reporting Intens for Systematic Rewiews and Meta-PRISMA (Galvão, Pansani, \& Harrad, 2015). Inicialmente, o levantamento dos artigos indexados foi realizado mediante a busca eletrônica em bases de dados, como: Biblioteca Virtual de Saúde - BVS, SCIELO, PERIODICOS CAPES e Education Resources Information Center - ERIC. A partir da relação das palavras-chave: Transtorno do Espectro Autista AND Homeschool, estratégias (adaptação). E, também, em inglês Disturb Espectrum Autism 
AND homeschool. As consultas foram realizadas em uma única etapa, no mês de maio de 2019 e a busca concluíu o período de Janeiro de 2009 a Dezembro de 2018, totalizando 10 anos.

Inicialmente, verificou-se os descritores (palavras-chave): Transtorno do Espectro Autista (Autism Spectrum Disorders), Homeschool e adaptação (sinônimo de estratégia). O descritor homeschool é um termo novo, não havendo sinônimos para uma maior busca na pesquisa. Depois, foi verificado a incidência dos artigos publicados em janeiro de 2009 a dezembro de 2018, em revistas indexadas na base de dados Biblioteca Virtual de Saúde (BVS), sobre o tema de pessoas com Transtorno do Espectro Autista e a educação em casa. Para tanto, foram utilizados os cruzamentos dos descritores (palavra-chave) relacionados ao tema: Autism Spectrum Disorders AND Homeschool, Autism Spectrum Disorders AND Homeschool AND Adaptation; e Transtorno do Espectro Autista AND homeschool. Para as revistas indexadas pela base de dados: Periodicos. Capes, foram utilizados os seguintes cruzamentos: Autism Spectrum Disorders AND Homeschool, Autism Spectrum Disorders AND Homeschool AND Adaptation. Já, para as revistas indexadas pelas bases de dados: Scielo e ERIC, o cruzamento de descrimento foi: Autism Spectrum Disorders AND Homeschool.

A amostra compreendeu as publicações de artigos indexados em periódicos, selecionados a partir da leitura dos resumos após terem seguidos os seguintes critérios de inclusão: a) veículos de publicação: optamos por periódicos indexados, pois são órgãos de maior divulgação e fácil acesso aos pesquisadores; b) ano de publicação: foram selecionados artigos entre janeiro/2009 a dezembro/2018, totalizando um período de 10 anos; c) idioma de publicação: foram selecionados os artigos nas línguas: portuguesa, espanhola e inglesa; e d) modalidade de produção científica: foram incluídos artigos da área de psicologia e educação. Em relação aos critérios de exclusão, temos: a) títulos duplicados; b) títulos que não apresentavam relação com os descritores (palavra-chave); e c) resumo (Tabela 1) 
Tabela 1 - Protocolo PRISMA

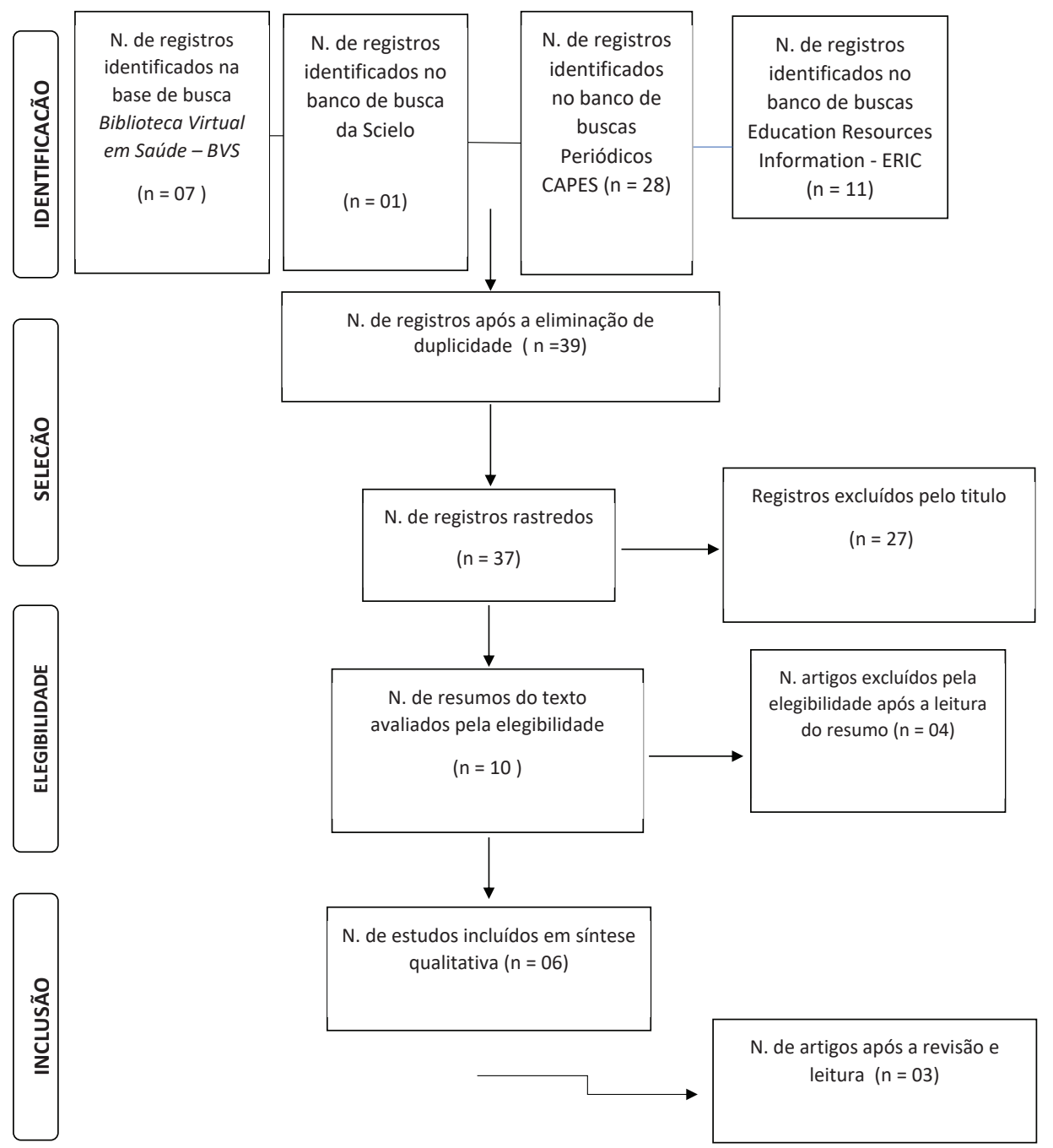

Fonte: Elaborada pela autora 
A estratégia inicial de busca dos artigos nas bases de dados referidos, totalizou 47 artigos. Primeiramente foi aplicado o critério de exclusão de artigos repetidos, totalizando 39 artigos. Com isto foi aplicado o critério de exclusão, de selecionar os artigos com o título que apresentasse um dos descritores, restando 10 artigos. Desses, houve a leitura crítica dos resumos, sendo eliminados 4 artigos que não preenchiam a resposta para a demanda dessa pesquisa. Desta forma, resultou-se em 6 artigos, conforme visualiza-se na Tabela 1.

Dos 6 artigos encontrados, apenas 5 foram recuperados e após a leitura minuciosa dos textos se verificou que dois não apresentam dados empíricos, mas dados para como se estabelecer um homeschool baseados em pesquisas, ficando 3 artigos. Todos os artigos utilizados estavam escritos na língua inglesa, e tinham como participantes pais de crianças com Transtorno do Espectro Autista que moravam nos Estados Unidos da América.

\section{Resultados}

Serão apresentados os resultados da revisão sistemática da literatura sobre o uso de pontos de benefícios do programa de Homeschool para as famílias e em seguida, os que marcam o fracasso desse programa. Assim, os 3 estudos selecionados de acordo com nossos critérios serão descritos e discutidos.

Conforme explicado, dentre os 3 artigos selecionados foi possível agrupar duas categorias visando uma melhor análise dos dados. Assim, temos:

\section{Benefícios}

Foi verificado que a decisão para que a família escolha o programa de homeschool para a criança com TEA é originada devido a preocupação dos pais de que o ambiente escolar e os laços sociais não estão preparados para receber essas crianças com comportamentos diferenciados. Assim, com o homeschool evitam que seus filhos sofram algum tipo de exclusão social e pela questão de não 


\begin{tabular}{|c|c|c|c|c|c|}
\hline Artigos & Títulos & Autores & Objetivo & Método & Ano \\
\hline 01 & $\begin{array}{l}\text { Experiences of } \\
\text { parentes who } \\
\text { homeschool their } \\
\text { children with } \\
\text { autismo spectrum } \\
\text { Disorders }\end{array}$ & $\begin{array}{l}\text { Karen S. } \\
\text { Hurlbutt }\end{array}$ & $\begin{array}{l}\text { Entrevistar } \\
\text { os pais que } \\
\text { colocaram seus } \\
\text { filhos com } \\
\text { transtorno do } \\
\text { Espectro Autista } \\
\text { no programa de } \\
\text { Homeschool. }\end{array}$ & $\begin{array}{l}\text { A pesquisadora } \\
\text { entrou em contato } \\
\text { com a delegacia de } \\
\text { ensino do Centro } \\
\text {-oeste dos E.U.A } \\
\text { para obter uma } \\
\text { lista das famílias } \\
\text { que participam } \\
\text { desse programa e } \\
\text { fez um convite a } \\
\text { cada família. Nove } \\
\text { aceitaram participar } \\
\text { e um casal resolveu } \\
\text { participar juntos, } \\
\text { totalizando } 10 \\
\text { entrevistados. } \\
\text { O Conselho de } \\
\text { revisão institucional } \\
\text { da Universidade } \\
\text { Estadual de } \\
\text { Minnesota, Mankato, } \\
\text { deu a aprovação } \\
\text { para o estudo a ser } \\
\text { conduzido. E os } \\
\text { participantes e seus } \\
\text { filhos receberam } \\
\text { pseudónimos. }\end{array}$ & 2011 \\
\hline 02 & $\begin{array}{l}\text { Considering } \\
\text { Homeschooling } \\
\text { Your Child } \\
\text { on the Autism } \\
\text { Spectrum? Some } \\
\text { Helpful Hints and } \\
\text { Suggestions for } \\
\text { Parents }\end{array}$ & $\begin{array}{l}\text { Karen S. } \\
\text { Hurlbutt }\end{array}$ & $\begin{array}{l}\text { Descrever e } \\
\text { considerar a } \\
\text { importância } \\
\text { de iniciar o } \\
\text { programa } \\
\text { Homeschooling }\end{array}$ & $\begin{array}{l}\text { Foi apresentando } \\
\text { questões sobre a } \\
\text { importância do } \\
\text { homeschool. }\end{array}$ & 2010 \\
\hline 03 & $\begin{array}{l}\text { Homeschool } \\
\text { Decision-Making } \\
\text { and Evidence- } \\
\text { Based Practice } \\
\text { for Children with } \\
\text { Autism Spectrum } \\
\text { Disorder }\end{array}$ & $\begin{array}{l}\text { Christina A. } \\
\text { Simmons\& } \\
\text { Jonathan M. } \\
\text { Campbell }\end{array}$ & $\begin{array}{l}\text { Entrevistar os } \\
\text { pais }\end{array}$ & $\begin{array}{l}\text { As entrevistas foram } \\
\text { conduzidas com } 9 \\
\text { parentes de crianças } \\
\text { com TEA e } \\
\text { do programa } \\
\text { Homeschooling } \\
\text { Cinco temas } \\
\text { distintos emergiram } \\
\text { em relação aos } \\
\text { motivos pelos quais } \\
\text { os pais decidiram } \\
\text { Homeschool para } \\
\text { seus filhos. }\end{array}$ & 2018 \\
\hline
\end{tabular}


terem ansiedade, depressão, necessidade de mais psicotrópicos, explosões, diminuição de autoconfiança e saúde comprometida, crença que tinham que o ambiente escolar causava (Simmons \& Campbell, 2018).

Esses mesmos autores também relatam que esta decisão sugere aos pais um alívio e diminuição do estresse noturno, pois não são mais obrigados a convencer todas as noites seus filhos que no outro dia terão que ir para escola.

Um outro fator apontado como benefício para a inserção do programa é de solucionar a insatisfação com a educação, porque os professores com salas de 20 a 25 alunos passaram a receber alunos com TEA, e não havia preparo para eles, tendo uma inadequação e muitos desentendimentos com os pais desses alunos pais optaram pela homeschool. E esses pais não precisavam mais: instruir os professores e diretores sobre leis e direitos das crianças com TEA; a sua casa teria mais condição de adaptação sensorial que a escola; e a socialização promovida pelos pais seria mais ampla (Hurlbutt, 2010).

A questão das interações chamadas negativas, como professores que não sabem a respeito do autismo e como lidar com as mudanças de comportamentos dessas crianças, fez com que os pais dessas pesquisas se afastassem da possibilidade de estar na escola.

Além do medo das influências das drogas, conflitos e possíveis lutas corporais que a criança com TEA ocasionava um maior isolamento social. Além da questão que quando agravo seu comportamento e por dificuldade essa criança era colocado numa sala com outras crianças com transtornos severos, havia uma identificação e imitação dos comportamentos, é isso se tornava frustrante e preocupante aos pais. Entretanto, Homeschooling não é para todos, mas pode ser a melhor escolha para aquela situação (Hurlbutt, 2011).

\section{Prejuízos}

A primeira questão que surge sobre essa categoria, diz respeito a escolha de vida, uma vez que influenciará a todos em casa, podendo repercutir no casamento, gerando conflitos e sérias discussões, pois 
o casal tem que estar sempre em harmonia para as decisões tomadas pela homeschool (Hurlbutt, 2010). Em 2011, essa mesma autora completa essa afirmação que havia um dado que $80 \%$ a $85 \%$ dos casais que aderiam a homeschool se separam, mas foi constatado que essa alta é um mito e que a separação ocorre em torno de $65 \%$.

Em 2010, Hurlbutt discute a ideia que a decisão de Homeschooling, não é apenas uma escolha educacional, mas uma escolha de estilo de vida, pois os pais enfrentaram questões de sobrevivência, tempo e esforço no seu casamento. Os pais devem concordar com os métodos e estratégias a serem usados em Homeschooling com o seu filho, sendo que os pais, por sua vez, já apresentam elevado nível de stress e preocupações por causa de ter um filho sobre o espectro do autismo, e o programa sugere um acréscimo de mais stress se não estiverem de acordo em conjunto.

Um outro fator evidenciado é sobre as questões financeiras, pois nessa escolha sempre um dos pais tem que parar de trabalhar ou se realiza home-office, acaba tendo um prejuízo devido a atenção que deve se dedicar ao homeschool (Simmons, \& Campbell, 2018)

Os referidos autores relatam que apesar da escolha de vida que decidem, acabam por entrar em um novo conflito, pois os pais além dessa função, percebem que não são professores, considerando a necessidade de contratar tutor ou professor particular, tendo um maior gasto financeiro. $\mathrm{Na}$ educação, passam a ter que testar seus filhos e não sabem como fazer, e percebem uma defasagem no conteúdo das matérias exigidas pelo governo de educação.

\section{Discussão}

É possível observar que a escolha do programa Homeschooling é baseado em sentimentos de frustrações e sobre o medo de exclusões que os filhos com TEA sofram na sociedade. Além disso, implica numa mudança de estilo de vida, a qual sugere que todo o grupo familiar esteja em harmonia nas decisões.

Tal prática se apresenta como análoga a outras características da sociedade dita "pós-moderna", à medida em que essas famílias 
se isolam e formam grupos independentes, sob a sua estrita responsabilidade, como a estruturação, ações, métodos, conteúdos e procedimentos do programa, ocasionando com que cada família seja exclusivamente a responsável pela educação de seus filhos.

Uma outra questão possível de ser levantada é a deficiência do sistema escolar, cada vez mais o modelo da escola obrigatória é colocado em questão e surgem formulações alternativas que permitem se pensar a educação fora da escola, ou seja, entre elas, reconhecer a casa, novamente, como espaço de educação.

Homeschooling ainda não é uma modalidade legalizada no Brasil e no mundo, e releva uma necessidade de aprimoramento ao sistema educacional e sociedade, apresentando muitas divergências e questões sobre a sua inserção. Entretanto, para as crianças autistas ainda releva um fator negativo que é a perda do convívio social e identificação com o semelhante que a escola deveria proporcionar.

A questão que apontada pelos resultados desta pesquisa de revisão sistemática é a necessidade da realização de pesquisa experimental para verificar a eficácia desse programa, pois os dados são de entrevistas de voluntárias e de pesquisa qualitativa, ou seja, os dados são pelas percepções de experiências individuas com os filhos que tem TEA (Simmons, \& Campbell, 2018).

\section{Considerações finais}

$\mathrm{O}$ autismo integra um cenário complexo que traz à tona uma problemática para a saúde pública. A idade apropriada para o início dos tratamentos é antes dos 3 anos, pois alguns sintomas ainda não se instauram e ainda é possível pensar na neuroplasticidade que facilita no desenvolvimento e melhor prognóstico para a criança. Assim, a prevenção dos traços autísticos em um bebê demonstra um progresso significativo e ambicioso para a saúde pública.

Em relação a educação é reconhecido que a inclusão se constitui em um fator importante para a construção da diversidade humana. Desta forma, o desafio se estabelece no aspecto coletivo, ou seja, uma sociedade sem escolas teria que buscar outras maneiras 
de promover a socialização dos sujeitos, além de ter que encontrar outras maneiras de atingir a todos, para ocasionar a pactuação social entre o estado e os indivíduos.

\section{Referências}

Blog da cidadania . (12 de 04 de 2019). Fonte: https://blogdacidadania.com. br/2019/04/damares-diz-que-pais-gostariam-que-filhos-com-deficiencia-nao-fossem-a-escola/

BRASIL. (06 de 06 de 2015). LEI Nº 13.146, DE 6 DE JULHO DE 2015. Acesso em 15 de 05 de 2019, disponível em Presidência da República: http://www. planalto.gov.br/CCIVIL_03/_Ato2015-2018/2015/Lei/L13146.htm

CDC. (2019). Fonte: Controle de Doenças e Prevenção de Saúde.

Chaves Vasconcelos, M. C., \& Bernadino Carvalho Morgado , J. C. JAN/ABR de 2014). Desafios à escolarização obrigatória: a inserção da homeschooling na legislação educacional no Brasil e em Portugal. RBPAE, 30, 203-230.

DSM-5. (2013). Fonte: American Psychiatric Association: goo.gl/qQPK8c

ENAD. (2019). https://aned.org.br/. Acesso em 14 de 06 de 2019, disponível em ENAD - ASSOCIAÇÃO NACIONAL DE EDUCAÇÃO DOMICILIAR.

Galvão, T. F., Pansani, T. d., \& Harrad, D. (2015). Principais itens para relatar Revisões sistemáticas e Meta-análises: A recomendação PRISMA. Epidemiologia e serviços de saúde. 24(2), 335-342. doi:10.5123/51679-49742015000200017

Hurlbutt, K. S. (2010). Considering homeschooling your child on the autism spectrum? some helpfull hints and suggestions for parents.

Hurlbutt, K. S. (2011). experiences of parents who homeschool their children with autism spectrum disorders. Focus on autism and other developmental disabilities, 26(4), 239-249. doi:10.1177/1088357611421170

Merlleti, C. (2018). Autismo em causa: historicidade, diagnóstico, prática clínica e narrativa dos pais. Stilos da clinica, 29, 149-151.

NCES, I. . (2019). https://nces.ed.gov/. Acesso em 14 de junho de 2019, disponível em National Center for Eduaction Statics. 
Portolese, J., Bordini, D., Lowenthal, R., Zachi, E. C., \& Silvestre de Paula , C. (2017). Mapeamento dos serviços que prestam atendimento a pessoas com transtornos do espectro autista. Caderno de pós - graduação em distúrbios do desenvolvimento, 17, pp. 79-91. doi:10.5935/cadernosdisturbios.v17n2p79-91

Ribeiro, A. M. (2011). O ensino doméstico e a organização esciolar: um contributo sociológico -organizacional sobre a realidade portuguesa. dissertação de mestrado instituto de ciências de educação, universidade de minho , Braga. Acesso em maio de 2019

Ribeiro, P. R. (jan/abr de 2006). Historia da saúde Mental Infantil: a criança brasileira da colônia à república velha. Psicologia em estudo, 11, 29-38.

Saúde, M. d. (2015). Acesso em 15 de 04 de 2019, disponível em ministério da Saúde. linha de cuidado para a atenção às pessoas com transtornos do espectro do autismo em suas na rede de atenção psicossocial do sistema ùnico de Saúde: www. saude.gov.br/saudemental

Simmons , C. A., \& Campbell, J. M. (27 de novembro de 2018). Homeschool Decision - making and evidence - Based practice for children with autism spectrum disorder. Journal of developmental and physical disabilities, 31, 329-346. doi:10.1007//s10882-018-9643-8

Vasconcelos , M. C., \& Morgado ,J. B. (2014). Desafios à escolarização obrigatória: a inserção do homeschooling na legislação educacional no Brasil e em Portugal. Revista Brasileira de Política e Administração da Educação, 30, 203-230. 